PROOF COPY [JTE20150186]

Journal of Testing and Evaluation

doi:10.1520/JTE20150186 / Vol.45 / No.4 / July 2017 / available online at www.astm.org

Manuscript received May 5, 2015; accepted for publication May 4, 2016; published online $x x$ xx xxxx.

${ }^{1}$ Dept. of Chemical and Environmental Engineering, University of Debrecen, 2-4 Ótemető St., Debrecen HU-4028, Hungary (Corresponding author), e-mail: kocsis.denes@eng.unideb.hu

2 Dept. of Applied Chemistry, Univ. of Debrecen, 1 Egyetem Square, Debrecen HU-4032, Hungary,

e-mail: deak.gyorgy@science.unideb.hu

${ }^{3}$ Dept. of Applied Chemistry, Univ. of Debrecen, 1 Egyetem Square, Debrecen HU-4032, Hungary,

e-mail: keki.sandor@science.unideb.hu

${ }^{4}$ Dept. of Information Technology, Univ. of Debrecen, 26 Kassai St., Debrecen HU-4028, Hungary,

e-mail: godo.zoltan@inf.unideb.hu

${ }^{5}$ Dept. of Chemical and Environmental Engineering, Univ. of Debrecen, 2-4 Ótemetö St., Debrecen HU-4028, Hungary, e-mail: horobjan@gmail.com
D. Kocsis, ${ }^{1}$ G. Deák, ${ }^{2}$ S. Kéki, ${ }^{3}$ Z. A. Godó, ${ }^{4}$ and R. Horváth ${ }^{5}$

\section{Creep and Quasi-Relaxation Examination of Artificially Aged Plasticized PVC}

\section{Reference}

Kocsis, D., Deák, G., Kéki, S., Godó, Z. A., and Horváth, R., "Creep and Quasi-Relaxation Examination of Artificially Aged Plasticized PVC," Journal of Testing and Evaluation, Vol. 45, No. 4, 2017, pp. 1-9, doi:10.1520/JTE20150186. ISSN 0090-3973

\section{ABSTRACT}

This paper introduces long-term tensile and quasi-relaxation tests of polyvinylchloride (PVC) fibers. During creep, the longitudinal and cross-sectional changes were measured of unaged and aged PVC fibers loaded with the same weight. In the quasi-relaxation section, where deformation was frozen, the stress changes were determined. During the examination of the creep, the Poynting-Thomson model described the phenomenon with sufficient accuracy ( $\min R^{2} 0.9628$ ) and the calculated parameter values characterized the aging process well. In the relaxed phase, the Poynting-Thomson model was not adequate, and, therefore, the second-order time derivatives were also involved in the applied model. Aging significantly resulted in parameter changes in this section as well.

\section{Keywords}

aging, creep, material property tests, PVC, relaxation

\section{Introduction}

The plastic products (heating pipes, tubes, barrier films, etc.) are typically planned for a long service life. Sometimes unexpected degradation events happen long before the end of the planned lifecycle (gas pipes crack, thermal water pipelines fracture, etc.). This can be the consequences of the degradation of plastics under external effects. Extensive knowledge of polymer degradation is required by many industrial applications [1]. The fate of outdoor aged polymers can be anticipated from accelerated laboratory tests; therefore, artificial aging experiments of plastics are considered to be important.

A number of material models try to describe the material behavior, from simple rheological models (Hooke, Newton, St. Venant) to complex material models. These can be formed as a 
combination of the simple models (e.g., Maxwell, Poynting -Thomson, Burgers). More sophisticated constitutive material models exist with the modification of the previous ones taking into account physical aspects of material internal dissipation $[2,3]$. This more precisely describes the material behavior, such as creep, relaxation, inertia, etc. Based on these models, exploring can be used for describing the material behavior accurately. In this case, this is done by determining its characteristic material parameters. Another aim is to identify the parameter changes caused by artificial aging, and to obtain information about their changing direction and magnitude.

The thermoplastic materials at small deformation behave as visco-elastic, and visco-plastic at high deformation. For the simulation of their behavior, a proper material model is necessary. There are widely used practical solutions for the simulation of thermoplastic materials, which are based on modifications to metal-based models. These models try to predict the behavior of thermoplastics. One is the semi-analytical model for polymers (SAMP) model introduced by Haufe et al. [4]. It considers the hydrostatic pressure dependence of thermoplastics' yield stress. Experimental studies are often based on modeling the creep and the stress relaxation together [5].

Recently, several material models have been formed. They try to predict the material behavior of thermoplastics by splitting the overall stress into different parts. In many cases, these models work with a different number of material parameters, such as those created by Krempl and Ho [6] for PA66 nylon, which had 15 parameters. Similar models were formed for high density polyethylene (HDPE) [7], polyoxymethylene (POM) [8], and polypropylene (PP) [9]. Nikolov and Doghri [10] presented a micromechanically based constitutive model for the small deformation behavior of HDPE. A method for modeling the nonlinear viscoelastic response of polymers was introduced by Joseph [11], with the comparison of model response and experimental data of different materials including polyvinylchloride (PVC). Pagnacco et al. [2] proposed a hybrid numerical/experimental approach to determine elasticity and viscoelasticity parameters of an isotropic material, and they demonstrated an application to a real rigid PVC plate. It is obvious that choosing the right number of parameters for an appropriate description of a material behavior is crucial. Yonan et al. [12] presented a nonlinear visco-plastic material model for PVC; a total of seven material parameters are necessary.

Numerous authors used fractional calculus for describing the properties of viscoelastic materials. Nonnenmacher and Glöckle [13] generalized the Poynting-Thomson model by applying the fractional calculus. Gloeckle and Nonnenmacher [14] presented an exactly solvable fractional model of linear viscoelastic behavior. Metzler and Nonnenmacher [15] investigated fractional relaxation processes and applied fractional rheological models for the description of viscoelastic materials. Mainardi and Spada [16] provided an overall survey to the viscoelastic models constructed via fractional calculus. Liu and 75 $\mathrm{Xu}$ [17] applied constitutive equations of viscoelastic materials 76 involving three different fractional parameters. They presented 77 the calculated material parameter values for the experimental 78 data of a viscoelastic material.

With regard to the known beneficial properties of PVC, a 80 number of earlier studies examined the property changes caused 81 by different aging processes. The most important factors influ- 82 encing degradation of PVC materials include oxygen, humidity, 83 mechanical stress, aggressive media, and ionizing radiation, all 84 being accelerated by increasing temperature [18]. PVC exposed 85 to weathering deteriorates and becomes increasingly colored 86 and brittle. This results in a continuous decrease in mechanical 87 properties such as tensile strength, elasticity, and impact resist- 88 ance [19]. Artificial aging is usually performed at a high temper- 89 ature, such as Yarahmadi et al. [20]. They studied the effects of 90 heat treatment on the mechanical properties of PVC. Zhou 91 et al. [21] investigated the creep performance of PVC aged at 92 high temperature. Barbero and Ford [22] examined physical 93 aging and temperature effects on PVC creep and relaxation. 94 They applied the equivalent time temperature method to deal 95 with long-term creep data. In addition, many studies work with 96 photodegradation tests, including Ito and Nagai [23] and 97 D'Aquino et al. [24]. D'Aquino uses a simplified mathematical 98 model to predict PVC photodegradation.

The aim of this work is to investigate and describe the 100 behavior of the examined material with sufficient precision 101 using the applied load at constant temperature, and to gain in- 102 formation about the aging process using this model. Therefore, 103 this work tries to minimize the number of necessary material 104 parameters, which are used to characterize the possible changes 105 and the level of degradation. 106

\section{Experimental}

TEST MATERIAL

Soft PVC fibers were used in the measurements, which were 109 extruded from LE 411 type granulate produced by BorsodChem 110 (Kazincbarcika, Hungary). Material composition is S-5070 PVC 111 ( $K=70) 60.30$ wt. \%; Bis(2-ethylhexyl) phthalate 37.39 wt. \%; 112 TM181-FSM stabilizer 0.90 wt. \%; MMA/EA 1.21 wt. \%; E-wax 113 0.18 wt. \%; and Uvitex OB fluorescent whitener 0.02 wt. \%. 114 Mechanical properties are hardness shore A 72; density 115 $1.217 \mathrm{~g} / \mathrm{cm}^{3}$; tensile strength $18.1 \mathrm{MPa}$; and elongation at break 116 $340 \%$. The diameters of the fibers were approximately $8 \mathrm{~mm}, 117$ and the examined length was $250 \mathrm{~mm}$.

\section{INSTRUMENTS}

The PVC fibers were exposed to UV light from Sylvania ultra- 120 violet G30W lamps (two lamps, ultraviolet radiation with a 121 peak at $253.7 \mathrm{~nm}$, illumination intensity: $2 \times 1720 \mathrm{~mW} / \mathrm{cm}^{2}, 122$ and the distance between the UV light and the PVC material: 123 
FIG. 1 Schematic view of the experimental system: (a) stretched PVC fiber, (b) load cell, (c) weight loaded on the specimen, and (d) pulley.

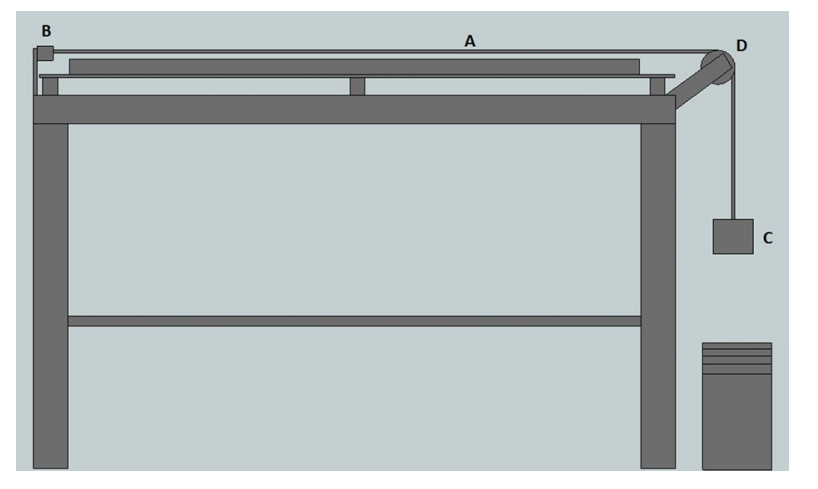

FIG. 2 Pictures of the experimental system

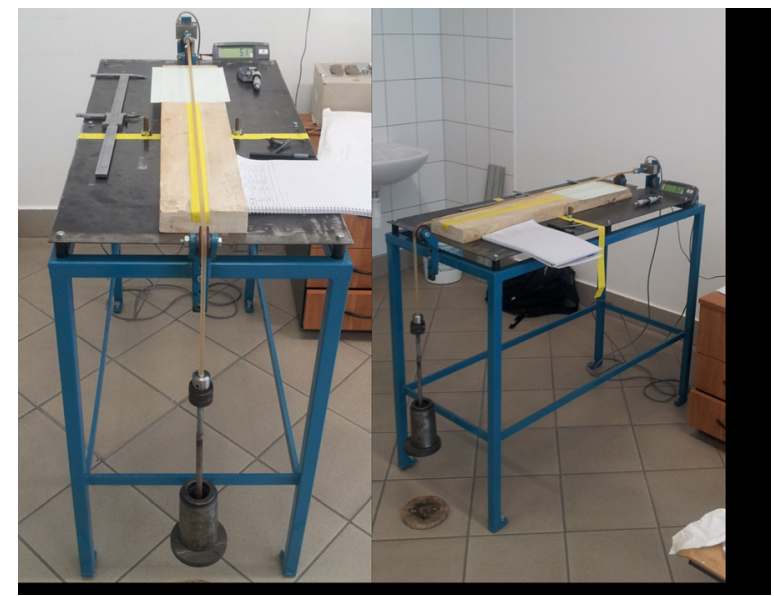

The weight loading of the specimen moved vertically down- 144 ward. In each case during this experiment, the weighted end 145 was stopped by placing blocks under it after a three-and-a-half 146 hour creep period. Thanks to this "setup," the further longitudi- 147 nal deformation was frozen. Then quasi-relaxation (not based 148 on standards like ASTM E328-13, ASTM D6048-07, etc.; here- 149 inafter named simply relaxation) was studied based on the load 150 cell's values and the longitudinal and transverse deformation. 151 AQ2 Finally, after removing the weight, the specimen was removed 152 from the table in an idle state. The length and diameter were 153 measured again at different times. Thus, the following stages 154 can be examined during and after the experiment (Table 1). 155

\section{Results and Discussion}

The examinations are based on the deviatoric and spherical 157 split, which is recently applied by several authors [12,26,27]. 158 The split of the stress tensor and deformation tensor can be 159 seen in the Appendix. A universal model introduced by Asszo- 160 nyi et al. [28] consists of the Poynting-Thomson body with an 161 additional inertial element. The authors called the resulting 162 model the Kluitenberg-Verhás body, which can be written after 163 splitting:

$$
\begin{gathered}
\boldsymbol{\sigma}^{d}+\tau^{d} \dot{\boldsymbol{\sigma}}^{d}=\alpha^{d} \boldsymbol{\varepsilon}^{d}+\beta^{d} \dot{\boldsymbol{\varepsilon}}^{d}+\gamma^{d} \ddot{\boldsymbol{\varepsilon}}^{d} \\
\boldsymbol{\sigma}^{s}+\tau^{s} \dot{\boldsymbol{\sigma}}^{s}=\alpha^{s} \boldsymbol{\varepsilon}^{s}+\beta^{s} \dot{\boldsymbol{\varepsilon}}^{s}+\gamma^{s} \ddot{\boldsymbol{\varepsilon}}^{s}
\end{gathered}
$$

It is apparent that, in this case, the stress first derivative and 165 deformation first and second derivative are included in the 166 equations. The following examines whether these parameters 167 are sufficient in number for the studied case.

Deviatoric and spherical split is applied, because in terms of 169 viscoelasticity the deviatoric and spherical parts behave inde- 170 pendently based on general mechanical-thermodynamical con- 171 siderations [28]. For example, in the case of a uniaxial process, 172 the complex, multi-parametric viscoelastic behavior of tensile 173 strength and deformation can be divided into two simpler, less 174 parametric behaviors. This split makes the fitting of the material 175 coefficients from experimental data much easier. Therefore, it is 176 advantageous both from a theoretical and an evaluating per- 177 spective. The investigation is continued with the analysis of the 178 deviatoric part. The creep and quasi-relaxation phases are 179

TABLE 1 Stages of the experiment.

\begin{tabular}{lc}
\hline Stage & Characteristic \\
\hline 1. Loading & Infinite fast $\left(\dot{\varepsilon}_{\|} \rightarrow \infty\right)$ \\
2. Creeping & Approximately $3.5 \mathrm{~h}$ \\
3. Relaxation & Min 70, max $430 \mathrm{~h}\left(\dot{\varepsilon}_{\|} \rightarrow 0\right)$ \\
4. Unloading & Removing the specimen $\left(\dot{\varepsilon}_{\|} \rightarrow-\infty\right)$ \\
5. Negative creeping & After removing $(\sigma=\dot{\sigma}=0)$ \\
\hline
\end{tabular}


managed separately. In all cases, the derivate values are obtained from fitted curves, and the parameters came from linear fitting.

\section{CREEP}

After the infinite fast loading, the creep phase lasted three and a half hours, and it is ended with putting blocks under the specimen's weighted end, the so-called "setup." The $\sigma^{d}$ values versus experimental time were calculated from the previously introduced split. Then a curve was fitted to the gathered measurement points by the following equation:

$$
y=y_{0}+A_{1} e^{\frac{-x}{t_{1}}}
$$

In this uniaxial tensile test, the specimen was subjected to a strain over a long time, and the stress values were calculated over time. The resulting stress versus experimental time data could be fitted with the Prony series for tensile relaxation with the following equation [29]:

$$
\sigma^{d}(t)=\sigma_{\infty}^{d}+\sum_{i=1}^{n} \sigma_{i}^{d} \frac{\frac{-t}{e^{t_{i}}}}{}
$$

where:

$\sigma_{\infty}^{d}=$ the long-term modulus, and

$\tau_{i}=$ the relaxation times.

The data was fitted with the equation, and determining the necessary number of parameters for a good prediction was important. In this examination, only $i=1$ for the creep phase was used, as can be seen in the equation above.

From the obtained curves, the stress derivatives were calculated. The same method was used to the deviatoric deformation $\left(\varepsilon^{d}\right)$ values and the derivatives.

$$
\varepsilon^{d}(t)=\varepsilon_{\infty}^{d}+\sum_{i=1}^{n} \varepsilon_{i}^{d} e^{\frac{-t}{\tau_{i}}}
$$

where:

$$
\varepsilon_{\infty}^{d}=\text { the long-term modulus, and }
$$

$\tau_{i}=$ the relaxation times.

It should be noted that, here, the $i=1$ value was used again; only one exponential provided appropriate results. The illustration of the fittings in the case of a specimen can be seen in Fig. 3.

The parameter values were obtained by linear fitting, and, as a result, it was found that in the creep phase besides the stress and deformation the use of their first derivative gives an accurate description. Therefore, during this period, higher derivatives are unnecessary in these experiments. The calculated parameter results for the two different aging series and for the unaged specimens can be seen in Table 2. Because of the degradation of PVC, $\tau^{d}$ and $\beta^{d}$ values decrease and $\alpha^{d}$ values increase versus aging time.

For checking the appropriateness of the results, a corrected sample standard deviation (SD) was calculated for each specimen using the measured and computed $\varepsilon^{d}$ values in the 222 measurement points. The time and $\varepsilon^{d}$ values and the calculated 223 $\mathrm{SD}$ in the case of one sample specimen can be seen in Table 3. 224 The values of SD for all specimens were between 0.0020 and 225 0.0031 . Fig. 4 shows the relationship between the measured 226 points and the parameter results.

The characteristic parameter values determined during the 228 creep period went under a significant change as the aging time 229 increases. For modeling the creep, a Poynting-Thomson model 230 proved to be adequate, as the second derivative's multiplication 231 factor $\left(\gamma^{d}\right)$ could be 0 and then the fitting still provides satisfac- 232 tory results.

The results with the frequently used marking is shown by 234 the following formula:

$$
\tau^{d}=\tau, \quad \alpha^{d}=2 G, \quad \beta^{d}=2 \eta, \quad \gamma^{d}=\theta
$$

Based on the marking above, the average values for the different 236 aging times can be seen in Table 4.

\section{QUASI-RELAXATION}

The relaxation after the creep section began with the "setup" of 239 the weighted end of the specimen. The length of the relaxation 240 was not the same for every specimen, in some cases up to $430 \mathrm{~h} .241$ Values obtained at the beginning of relaxation were not taken 242 into account in the calculation because of the initial uncertainty. 243 During relaxation, the longitudinal and transverse deformations 244 were frozen, so the deformation was constant, and there is no 245 need to take the derivatives into consideration. Therefore, only 246 the deviatoric stress $\left(\sigma^{d}\right)$ versus time points were used for fit- 247 ting. In this case, one exponential was not sufficient; thus, two 248 exponentials were used here (Fig. 5):

$$
y=y_{0}+A_{1} e^{\frac{-x}{t_{1}}}+A_{2} e^{\frac{-x}{L_{2}}}
$$

As can be seen in Fig. 5, the one exponential fitting does not fol- 250 low the changes in the early phase of the relaxation; therefore, 251 two exponential approximations were applied in this case. This 252 equation is similar to the previously introduced Prony series, 253 with a value of $i=2$ :

$$
\sigma^{d}(t)=\sigma_{\infty}^{d}+\sum_{i=1}^{n} \sigma_{i}^{d} e^{\frac{-t}{\tau_{i}}}=\sigma_{\infty}^{d}+\sigma_{1}^{d} e^{\frac{-t}{\tau_{1}}}+\sigma_{2}^{d} e^{\frac{-t}{t_{2}}}
$$

where:

$\sigma_{\infty}^{d}=$ the long-term modulus again, and

$\tau_{i}=$ the relaxation times.

Similarly to the creep part, the deviatoric stress derivatives 258 were obtained from the curves. Then linear fitting was applied 259 to determine the parameters; among them, the second deriva- 260 tives were also necessary in this case. That means the previously 261 introduced Kluitenberg-Verhás model is not appropriate for 262 simulating the examined material's relaxation. The general 263 
FIG. 3

One and two exponential curve fitting in the case of an aged specimen (aged2_2): $R^{2}$ values, one exponential $0.9467\left(\sigma^{\alpha}\right)$ and $0.9746\left(\varepsilon^{d}\right)$; and two exponential $0.9566\left(\sigma^{\alpha}\right)$ and $0.9933\left(\varepsilon^{d}\right)$.

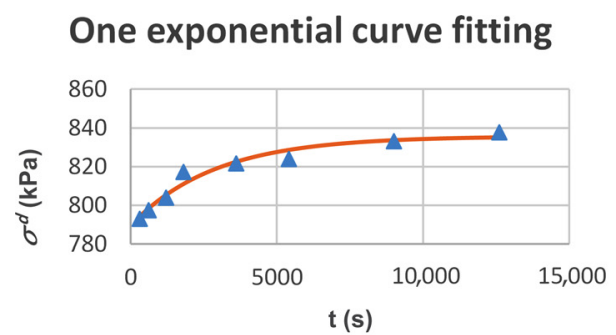

\section{Two exponential curve fitting}
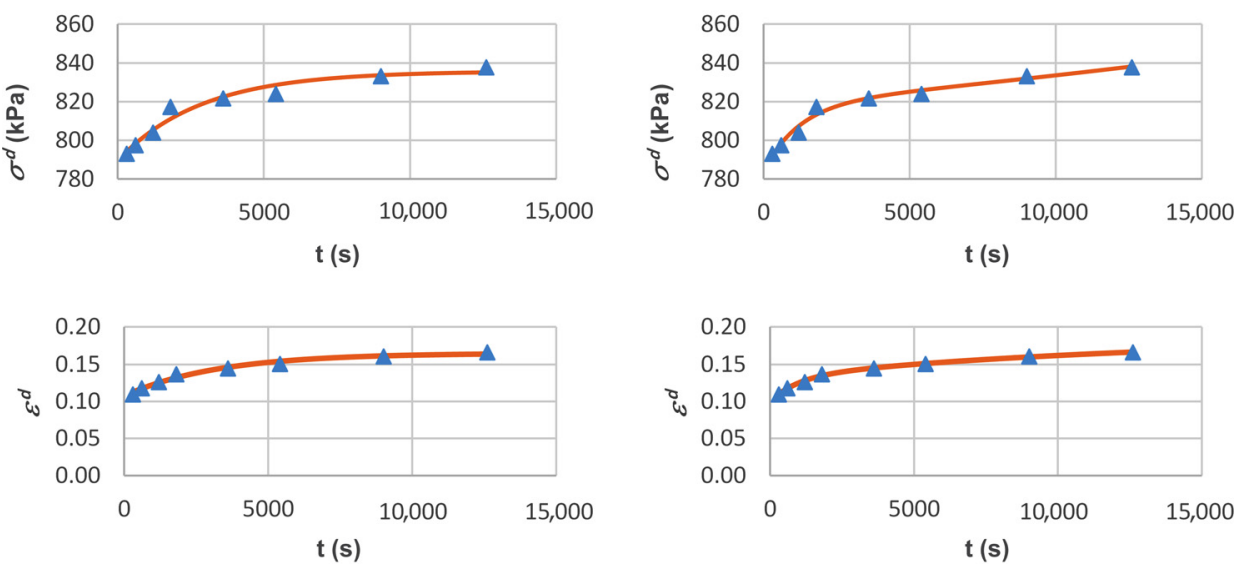

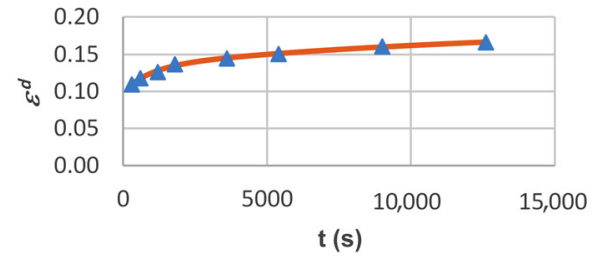

equation has to be expanded with the second derivative of stress and its factor:

$$
\sigma^{d}+\tau^{d} \dot{\sigma}^{d}+\xi^{d} \ddot{\sigma}^{d}=\alpha^{d} \varepsilon^{d}+\beta^{d} \dot{\varepsilon}^{d}+\gamma^{d} \ddot{\varepsilon}^{d}
$$

It should be noted that, in the creep period, only the first derivative of deformation and stress was taken into account. But here, a second derivative of stress was also necessary for appropriate characterization. The second derivative's multiplication coefficient $\left(\xi^{d}\right)$ is called inertial factor using the analogy of the differential equation of vibration. The application of the second derivative occurred in other models, such as the Burgers model. The obtained equation modifies the Burgers model where the zero-order derivate of epsilon is not included. Therefore, this model can be called a modified Burgers model. The calculated parameters can be seen in Table 5. Because of the degradation of PVC, $\tau^{d}$ and $\xi^{d}$ values decrease and $\alpha^{d}$ values increase versus aging time.

SD values were also calculated for checking the results of relaxation, but this time for the measured and calculated $\sigma^{d}$.

TABLE 2 Calculated parameters for creep section.

\begin{tabular}{lcccc}
\hline Aging Time $(\mathrm{h})$ & Specimen & $\tau^{d}(\mathrm{~h})$ & $\alpha^{d}(\mathrm{MPa})$ & $\beta^{d}(\mathrm{MPa}-\mathrm{h})$ \\
\hline 0 & 01 & 0.99 & 4.51 & 3.93 \\
& 02 & 1.65 & 4.48 & 4.63 \\
& 03 & 1.50 & 4.30 & 4.15 \\
\multirow{4}{*}{2541} & Average & 1.38 & 4.43 & 4.24 \\
& Aged1_1 & 0.66 & 5.00 & 3.16 \\
& Aged1_2 & 1.17 & 4.58 & 4.05 \\
& Aged1_3 & 0.95 & 4.40 & 4.52 \\
4000 & Average & 0.93 & 4.66 & 3.91 \\
& Aged2_1 & 0.29 & 5.90 & 3.52 \\
& Aged2_2 & 0.80 & 5.06 & 4.57 \\
& Aged2_3 & 0.52 & 5.13 & 3.35 \\
& Average & 0.54 & 5.36 & 3.81 \\
\hline \multirow{4}{*}{} & & & &
\end{tabular}

The SD for one sample specimen can be seen in Table 6, and in 281 every case SD values were between 3.7670 and 6.7958. Fig. 6282 shows the connection between the measurement points and the 283 calculated results.

In the case of relaxation, the determination of parameters 285 were always performed for the same interval (24,000-250,000 s). 286 But as seen in Fig. 6, the obtained parameter values well charac- 287 terize the overall relaxation. The obtained fitted parameters are 288 stable over the whole range. The results in the previously intro- 289 duced form is shown in Table 7.

Conclusions

In these experiments, long-term tensile and quasi-relaxation 292 tests were carried out on PVC fibers artificially aged by UV light 293 for different aging times. During the evaluation of the measure- 294 ment results, the creep and relaxation properties of the same 295 material were examined separately. The parameters were deter- 296 mined by fitting for the deviatoric parts of the stress tensor and 297 the deformation tensor. It was found for the creep phase that, in 298 the examined case, the changes can be described by the 299

TABLE 3 Calculated SD for a specimen (aged1_1).

\begin{tabular}{lcc}
\hline$t(\mathrm{~s})$ & Measurement $\varepsilon^{d}$ Values & Model $\varepsilon^{d}$ Values \\
\hline 300 & 0.1276 & 0.1323 \\
660 & 0.1405 & 0.1378 \\
1620 & 0.1480 & 0.1454 \\
1800 & 0.1512 & 0.1507 \\
3600 & 0.1592 & 0.1618 \\
5400 & 0.1651 & 0.1669 \\
9000 & 0.1713 & 0.1704 \\
12,600 & 0.1727 & 0.1711 \\
SD & & 0.0027 \\
\end{tabular}


PROOF COPY [JTE20150186]

FIG. 4 Calculated curves from the determined parameters and the measurement points (creep) min, $R^{2} 0.9628$.

Specimen 01

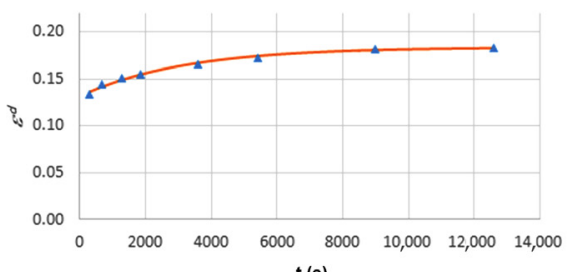

$\mathrm{t}$ (s)

Specimen aged1_1

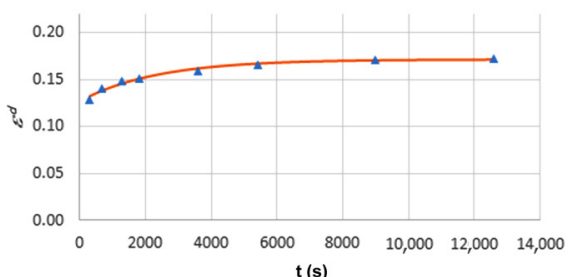

Specimen aged2_1

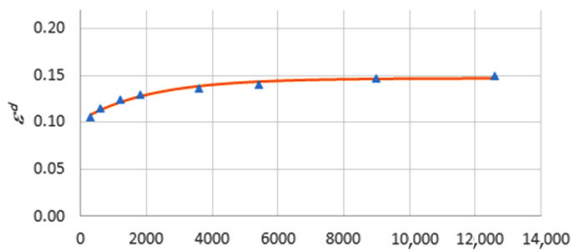

$t(s)$
Specimen 02

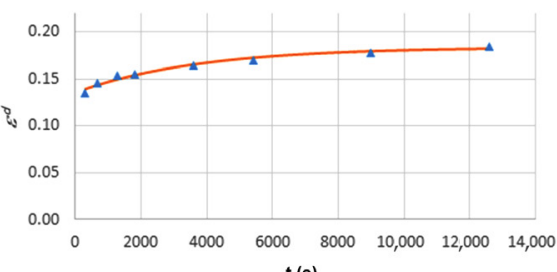

Specimen aged1_2

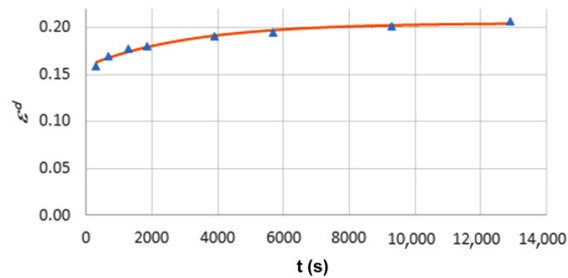

Specimen aged2_2

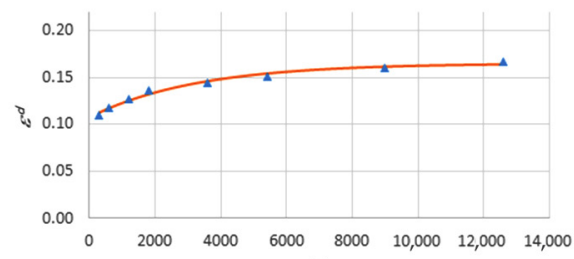

$t(s)$

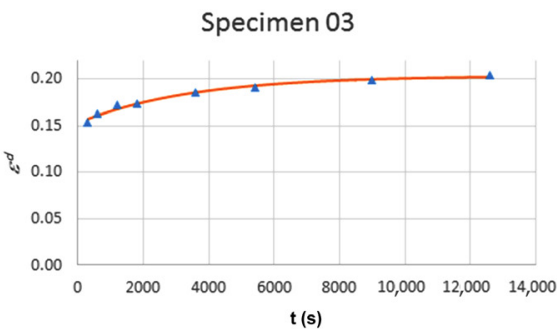

Specimen aged1_3

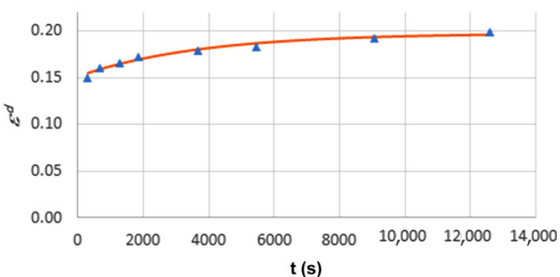

Specimen aged2_3

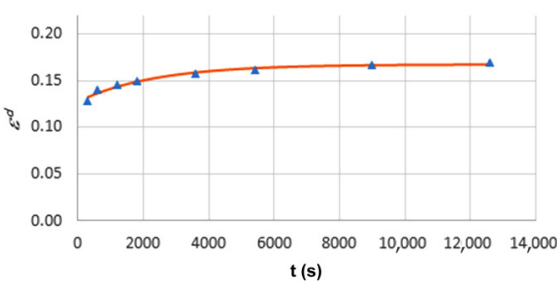

TABLE 4 Calculated average parameter values for creep section.

\begin{tabular}{lccc}
\hline Aging Time $(\mathrm{h})$ & $\tau(\mathrm{h})$ & $G(\mathrm{MPa})$ & $\eta(\mathrm{MPah})$ \\
\hline 0 & 1.38 & 2.22 & 2.12 \\
2541 & 0.93 & 2.33 & 1.95 \\
4000 & 0.54 & 2.68 & 1.95 \\
\hline
\end{tabular}

Poynting-Thomson model, and the resulting parameter values 300 well characterize the aging.

During the examination of relaxation, it was found that 302 using the second derivatives are also necessary for the suffi- 303 ciently accurate determination of stable parameters. Thus, in 304 this case, a modified Burgers model was applied, and the 305

\section{FIG. 5}

One and two exponential curve fitting with zooming in on their first part (specimen aged2_2): $R^{2}$ values, one exponential 0.9625 ; and two exponential 0.9933 .

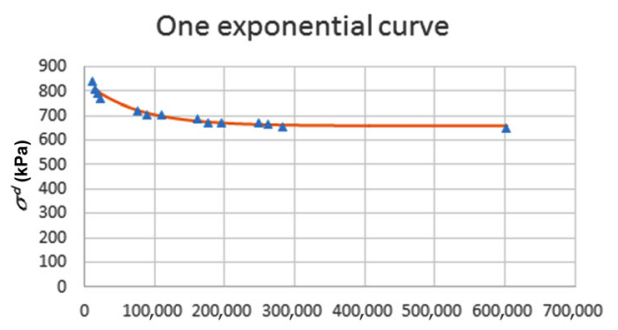

$t(s)$

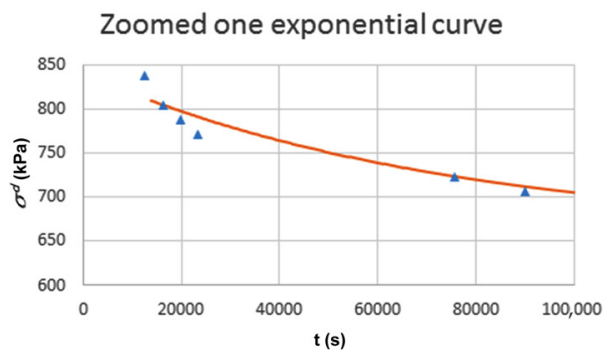

Two exponential curve

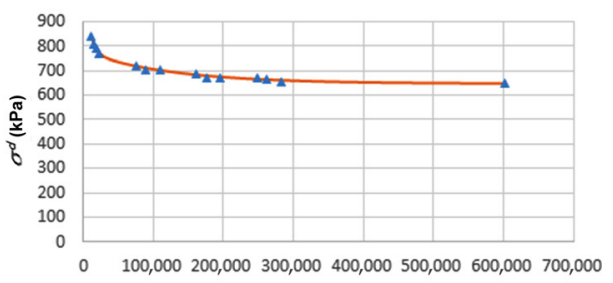

t (s)

\section{Zoomed two exponential curve}

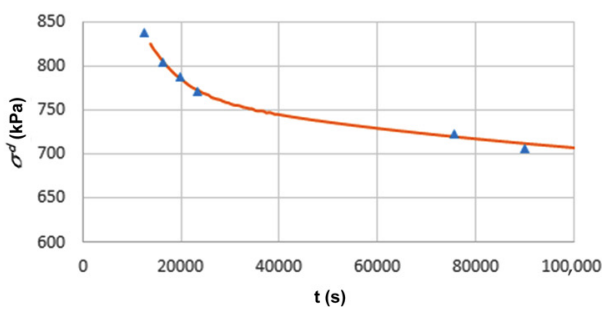

ID: asme3b2server Time: 13:55 I Path://chenas03/Cenpro/ApplicationFiles/Journals/ASTM/JTE\#/Vol04504/160068/Comp/APPFile/AT-JTE\#160068 
TABLE 5 Calculated parameters for quasi-relaxation section.

\begin{tabular}{lcccc}
\hline Aging Time $(\mathrm{h})$ & Specimen & $\tau^{d}(\mathrm{~h})$ & $\xi^{d}\left(\mathrm{~h}^{2}\right)$ & $\alpha^{d}(\mathrm{MPa})$ \\
\hline 0 & 01 & 70.32 & 327.82 & 3.07 \\
& 02 & 168.89 & 742.49 & 2.63 \\
& 03 & 192.40 & 706.29 & 2.76 \\
\multirow{4}{*}{2541} & Average & 143.87 & 592.20 & 2.82 \\
& Aged1_1 & 129.63 & 848.30 & 3.24 \\
& Aged1_2 & 80.09 & 328.13 & 3.10 \\
& Aged1_3 & 92.54 & 525.50 & 3.14 \\
4000 & Average & 100.75 & 567.31 & 3.16 \\
& Aged2_1 & 36.80 & 67.70 & 4.50 \\
& Aged2_2 & 37.37 & 71.77 & 3.89 \\
& Aged2_3 & 32.30 & 37.07 & 3.85 \\
& Average & 35.49 & 58.85 & 4.08 \\
\hline
\end{tabular}

TABLE 6 Calculated SD for a specimen (aged1_2).

\begin{tabular}{lcc}
\hline$t(\mathrm{~s})$ & Measurement $\sigma^{d}$ Values & Model $\sigma^{d}$ Values \\
\hline 23,700 & 865.76 & 868.37 \\
30,900 & 847.34 & 846.73 \\
77,700 & 792.08 & 790.82 \\
84,900 & 792.08 & 786.44 \\
92,100 & 773.66 & 782.35 \\
102,900 & 773.66 & 776.63 \\
110,100 & 773.66 & 773.02 \\
117,300 & 773.66 & 769.54 \\
164,100 & 755.24 & 749.31 \\
178,500 & 736.82 & 743.78 \\
200,100 & 736.82 & 736.01 \\
255,900 & 718.40 & 718.59 \\
SD & \multicolumn{2}{c}{} \\
\hline
\end{tabular}

FIG. 6 Calculated curves from the determined parameters and the measurement points (quasi-relaxation) min, $R^{2} 0.9720$.

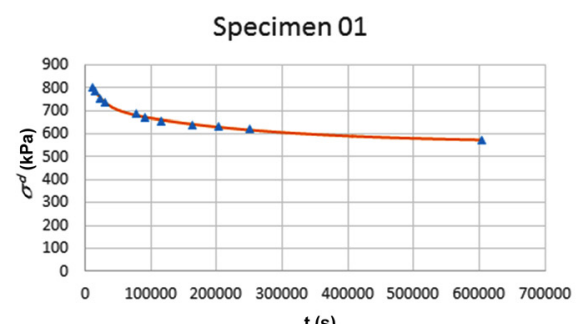

$t(s)$

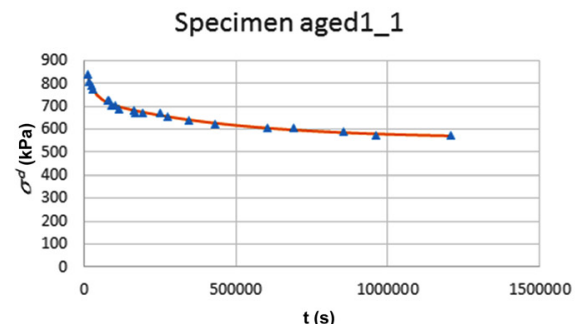

Specimen aged2_1

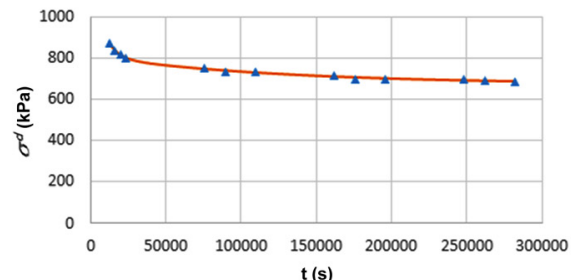

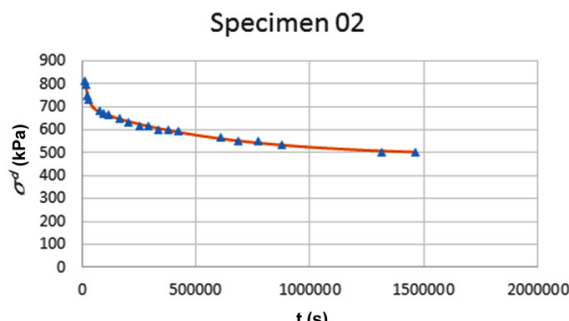

Specimen aged1_2

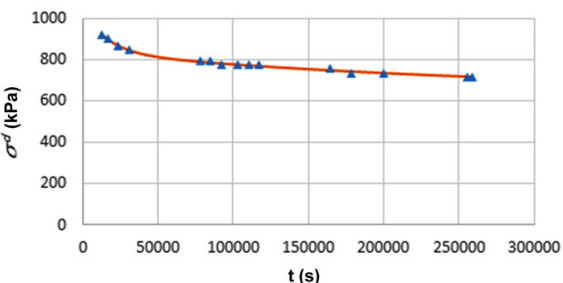

Specimen aged2_2

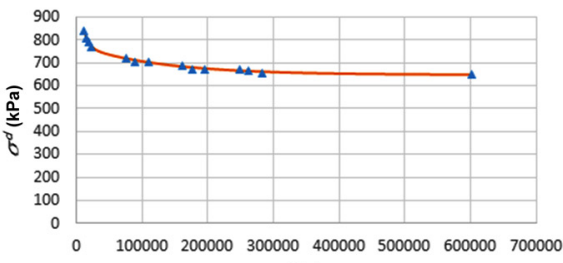

$t(s)$

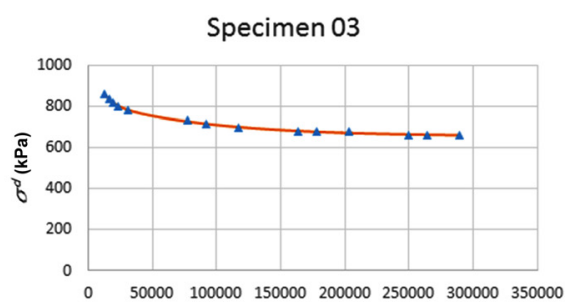

$t$ (s)

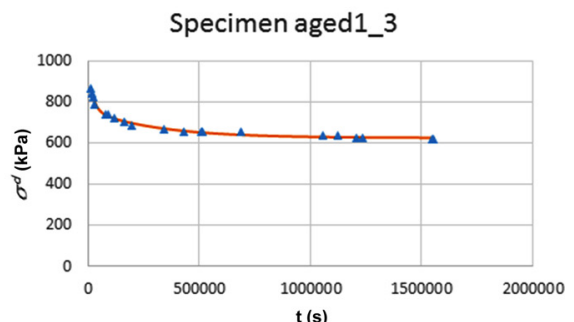

Specimen aged2_3

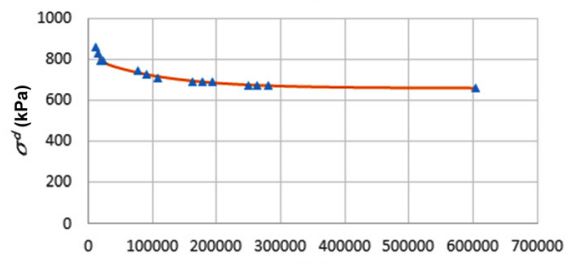

TABLE 7 Calculated average parameter values for quasi-relaxation section.

\begin{tabular}{lccc}
\hline Aging Time $(\mathrm{h})$ & $\tau(\mathrm{h})$ & $\xi^{d}\left(\mathrm{~h}^{2}\right)$ & $G(\mathrm{MPa})$ \\
\hline 0 & 143.87 & 592.20 & 1.41 \\
2541 & 100.75 & 567.31 & 1.58 \\
4000 & 35.49 & 58.85 & 2.04 \\
\hline
\end{tabular}

resulting relaxation parameters are proven to be very stable and 306 they well characterize the aging. Therefore, compared to other 307 methods mentioned in the introduction, the applied method 308 provides good results without fractional calculus or using a high 309 number of parameters. 310

This work contributes to permanent literature by introduc- 311 ing a new, modified Burgers model for the relaxation behavior 312 of PVC. The application of this model to other thermoplastic 313 


$$
\begin{aligned}
{\left[\mathbf{S}^{D}\right] } & =\left(\begin{array}{ccc}
\frac{2}{3} \sigma & 0 & 0 \\
0 & -\frac{1}{3} \sigma & 0 \\
0 & 0 & -\frac{1}{3} \sigma
\end{array}\right)=\left(\begin{array}{ccc}
\sigma^{D} & 0 & 0 \\
0 & -\frac{1}{2} \sigma^{D} & 0 \\
0 & 0 & -\frac{1}{2} \sigma^{D}
\end{array}\right) \\
{\left[\mathbf{S}^{V}\right] } & =\left(\begin{array}{ccc}
\frac{1}{3} \sigma & 0 & 0 \\
0 & \frac{1}{3} \sigma & 0 \\
0 & 0 & \frac{1}{3} \sigma
\end{array}\right)=\frac{1}{3}\left(\begin{array}{ccc}
\sigma^{V} & 0 & 0 \\
0 & \sigma^{V} & 0 \\
0 & 0 & \sigma^{V}
\end{array}\right) \\
{\left[\mathbf{E}^{D}\right] } & =\left(\begin{array}{ccc}
\frac{2}{3}\left(\varepsilon-\varepsilon_{T}\right) & 0 \\
0 & -\frac{1}{3}\left(\varepsilon-\varepsilon_{T}\right) & 0 \\
0 & 0 & -\frac{1}{3}\left(\varepsilon-\varepsilon_{T}\right)
\end{array}\right) \\
& =\left(\begin{array}{ccc}
\varepsilon^{D} & 0 & 0 \\
0 & -\frac{1}{2} \varepsilon^{D} & 0 \\
0 & 0 & -\frac{1}{2} \varepsilon^{D}
\end{array}\right) \\
& =\frac{1}{3}\left(\begin{array}{ccc}
\frac{1}{3}\left(\varepsilon+2 \varepsilon_{T}\right) & 0 \\
0 & \frac{1}{3}\left(\varepsilon+2 \varepsilon_{T}\right) & 0 \\
0 & 0 & 0 \\
0 & 0 & 0 \\
\varepsilon^{V} & 0 \\
0 & \varepsilon^{V}\left(\varepsilon+2 \varepsilon_{T}\right)
\end{array}\right)
\end{aligned}
$$

$$
\varepsilon=\text { the longitudinal, and }
$$$$
\varepsilon_{T}=\text { the transversal strain. }
$$

The stress tensor and deformation tensor can be split into deviatoric (marked with $D$ ) and spherical (or volumetric) parts (marked with $V$ ):

\section{References}

[1] Mahadevan, R. and Smith, L., "A Mechanistic Model Describing the Degradation of Polymers," J. Polym. Environ., Vol. 15, No. 2, 2007, pp. 75-80.

[2] Pagnacco, E., Moreau, A., and Lemosse, D., "Inverse Strat- 329 egies for the Identification of Elastic and Viscoelastic Mate- 330 rial Parameters Using Full-Field Measurements," Mater. 331 Sci. Eng. A-Struct., Vol. 452, 2007, pp. 737-745.

[3] Atanackovic, T. M., "A Modified Zener Model of a Visco- 333 elastic Body," Continuum Mech. Therm., Vol. 14, No. 2, 334 2002, pp. 137-148.

[4] Haufe, A., Du Bois, P. A., Kolling, S., and Feucht, M., “A 336 Semi-Analytical Model for Polymers Subjected to 337 High Strain Rates," 5th European LS-DYNA Users 338 Conference-Material Technology, Birmingham, U.K., 339 2005, pp. 2b-58.

[5] Sahu, R., Patra, K., and Szpunar, J., "Experimental Study 341 and Numerical Modelling of Creep and Stress Relaxation 342 of Dielectric Elastomers," Strain, Vol. 51, No. 1, 2015, 343 pp. 43-54.

[6] Krempl, E., and Ho, K., "An Overstress Model for Solid 345 Polymer Deformation Behavior Applied to Nylon 66," 346 Time Dependent and Nonlinear Effects in Polymers and 347 Composites, ASTM STP 1357, R. A. Schapery, Ed., ASTM 348 International, West Conshohocken, PA, 2000, 349 pp. 118-137, 350

[7] Colak, O. U. and Dusunceli, N., "Modeling Viscoelastic and 351 Viscoplastic Behavior of High Density Polyethylene 352 (HDPE)," J. Eng. Mater., Vol. 128, No. 4, 2006, pp. 572-578. 353

[8] Hartmann, S., "A Thermomechanically Consistent 354 Constitutive Model for Polyoxymethylene-Experiments, 355 Material Modelling and Computation," Arch. Appl. Mech., 356 Vol. 76, No. 5, 2006, pp. 349-366. 357

[9] Kästner, M., Blobel, S., Obst, M., Thielsch, K., and 358 Ulbricht, V., "Experimental Characterization of the Visco- 359 plastic Material Behaviour of Thermosets and 360 Thermoplastics," Appl. Mech. Mater., Vols. 24-25, 2010, 361 pp. 195-200.

[10] Nikolov, S. and Doghri, I., "A Micro/Macro Constitutive 363 Model for the Small-Deformation Behavior of Poly- 364 ethylene," Polymer, Vol. 41, No. 5, 2000, pp. 1883-1891. 365

[11] Joseph, S. H., "A Method for Modelling the Nonlinear 366 Viscoelastic Response of Polymers," Mech. Time-Depend. 367 Mater., Vol. 9, No. 1, 2005, pp. 35-69.

[12] Yonan, S. A., Soyarslan, C., Haupt, P., Kwiatkowski, L., 369 and Tekkaya, A. E., "A Simple Finite Strain Non-Linear 370 Visco-Plastic Model for Thermoplastics and Its Applica- 371 tion to the Simulation of Incremental Cold Forming of 372 Polyvinylchloride (PVC)," Int. J. Mech. Sci., Vol. 66, 2013, 373 pp. 192-201.

[13] Nonnenmacher, T. and Glöckle, W., "A Fractional Model 375 for Mechanical Stress Relaxation,” Philos. Mag. Lett., Vol. 376 64, No. 2, 1991, pp. 89-93.

[14] Gloeckle, W. G. and Nonnenmacher, T. F., "Fractional In- 378 tegral Operators and Fox Functions in the Theory of 379 Viscoelasticity," Macromolecules, Vol. 24, 1991, 380 pp. 6426-6434.

[15] Metzler, R. and Nonnenmacher, T. F., "Fractional Relaxa- 382 tion Processes and Fractional Rheological Models for the 383 Description of a Class of Viscoelastic Materials," Int. J. 384 Plast., Vol. 19, 2003, pp. 941-959.

[16] Mainardi, F. and Spada, G., "Creep, Relaxation and Viscos- 386 ity Properties for Basic Fractional Models in Rheology," 387 Eur. Phys. J. Special Topics, Vol. 193, No. 1, 2011, 388 pp. 133-160. 
[17] Liu, J. G. and Xu, M. Y., "Higher-Order Fractional Constitutive Equations of Viscoelastic Materials Involving Three Different Parameters and Their Relaxation and Creep Functions," Mech. Time-Depend. Mater., Vol. 10, No. 4, 2006, pp. 263-279.

[18] Jakubowicz, I., "Effects of Artificial and Natural Ageing on Impact-Modified Poly(Vinyl Chloride) (PVC)," Polym. Test., Vol. 20, No. 5, 2001, pp. 545-551.

[19] Feldman, D., "Polymer Weathering: Photo-Oxidation," J. Polym. Environ., Vol. 10, No. 4, 2002, pp. 163-173.

[20] Yarahmadi, N., Jakubowicz, I., and Hjertberg, T., "The Effects of Heat Treatment and Ageing on the Mechanical Properties of Rigid PVC," Polym. Degrad. Stab., Vol. 82, No. 1, 2003, pp. 59-72.

[21] Zhou, Z. H., He, Y. L., Hu, H. J., Zhao, F., and Zhang, X. L., "Creep Performance of PVC Aged at Temperature Relatively Close to Glass Transition Temperature," Appl. Math. Mech.-Engl., Vol. 33, No. 9, 2012, pp. 1129-1136.

[22] Barbero, E. J. and Ford, K. J., "Equivalent Time Temperature Model for Physical Aging and Temperature Effects on Polymer Creep and Relaxation," J. Eng. Mater. Technol., Vol. 126, No. 4, 2004, pp. 413-419.

[23] Ito, M. and Nagai, K., "Analysis of Degradation Mechanism of Plasticized PVC Under Artificial Aging Conditions," Polym. Degrad. Stabil., Vol. 92, No. 2, 2007, pp. 260-270.
[24] D’Aquino, C. A., Balmant, W., Ribeiro, R. L. L., Munaro, 416 M., Vargas, J. V. C., and Amico, S. C., "A Simplified 417 Mathematical Model to Predict PVC Photodegradation in 418 Photobioreactors," Polym. Test., Vol. 31, No. 5, 2012, 419 pp. 638-644.

[25] Devasenapathi, V., Monish, P., and Prabu, S. B., 421 "Experimental Investigation of Tensile Creep Behavior of 422 Polymer Nanocomposites," Int. J. Adv. Manuf. Tech., Vol. 423 44, No. 3, 2009, pp. 412-418.

[26] Ván, P., and Szarka, Z., "Rock Rheology-Time Depend- 425 ence of Dilation and Stress Arround a Tunnel," Eurock, 426 2006, Multiphysics Coupling and Long Term Behaviour in 427 Rock Mechanics, A. van Cotthem, R. Charlier, J. Thimus, 428 and J. Tshibangu, Eds., Taylor \& Francis, London, 2006, 429 p. 357.

[27] Fülöp, T., and Béda, G., "Rheological Dynamics of 431 Tunnels-An Analytical Investigation," Rock 432 Engineering in Difficult Ground Conditions: Soft Rocks 433 and Karst, I. Vrkljan, Ed., Taylor \& Francis, London, 434 2010, p. 441.

[28] Asszonyi, C., Fülöp, T., and Ván, P., "Distinguished Rheo- 436 logical Models for Solids in the Framework of a Thermo- 437 dynamical Internal Variable Theory," Continuum Mech. 438 Thermodyn., Vol. 27, No. 6, 2015, pp. 971-986.

[29] Barbero, E. J., Finite Element Analysis of Composite Materi- 440 als, CRC, Boca Raton, FL, 2007. 